\title{
UAV-gestützte Vermessung im Bergbau - Zur Frage der Genauigkeit unter Verwendung von Structure from Motion
}

\author{
Alexander Tscharf \\ Lehrstuhl für Bergbaukunde, Bergtechnik und Bergwirtschaft, Department Mineral Resources Engineering, \\ Montanuniversität Leoben, Leoben, Österreich
}

Eingegangen 17. April 2020; angenommen 20. April 2020; online publiziert 6. Mai 2020

\begin{abstract}
Zusammenfassung: Aufgrund immer schneller fortschreitender Betriebe und aufgrund des hohen personellen und zeitlichen Aufwands bei herkömmlichen Vermessungsmethoden bilden UAV-gestützte (unmanned aerial vehicle) photogrammetrische Verfahren schon seit einiger Zeit eine wertvolle Ergänzung zur Erfüllung der markscheiderischen Aufgaben im Tagbau. Obwohl vergleichbare Systeme bereits in der täglichen Praxis angekommen sind, sind die erreichbare Genauigkeit und insbesondere die genauigkeitswirksamen Einflussgrößen noch nicht abschließend untersucht, weshalb die im Beitrag präsentierte Dissertation auf genau diese Frage abzielt.

Zur Beantwortung der aufgeworfenen Fragen bilden neben theoretischen Analysen vor allem praktische Untersuchungen im Labor- und Realmaßstab den Kern der Forschungsarbeiten. Die Ergebnisse der Untersuchungen zeigen, dass bei „richtigem“ Einsatz (Systemdesign, Flugplanung und Auswertung) die Genauigkeitsanforderungen der Markscheideverordnung 2013 eingehalten werden können. Hinsichtlich Parameteridentifikation sind vor allem die signifikanten Einflüsse der Flughöhe, der Bildüberlappung und der Orientierung der Aufnahmeachse relativ zum Objekt zu nennen, wobei die gesteigerte Genauigkeit in unmittelbarem Zusammenhang zur Stabilität der Bildverknüpfung bzw. der Anzahl an gültigen Verknüpfungspunkten zu stehen scheint.
\end{abstract}

Schlüsselwörter: Markscheidewesen, UAV-Vermessung, Photogrammetrie, Structure from Motion,

Genauigkeitsuntersuchungen
UAV-based Mine Surveying-On the Question of Accuracy Using Structure from Motion

Abstract: Due to the fact that operations are progressing faster and faster and due to high personnel and time expenditure for conventional surveying methods, UAV-based (unmanned aerial vehicle) photogrammetric methods have been a valuable addition for some time now. Although comparable systems have been well established in practice, the achievable accuracy and, in particular, the accuracy-influencing factors have not yet been finally investigated, which is why the presented thesis aims at precisely this question.

In addition to theoretical analyzes practical research in laboratory and real-scale forms the core of the research work to answer the questions raised. The results of the investigations show that the "correct" use (system design, flight planning and processing) can comply with the accuracy requirements of Markscheideverordnung 2013. With regard to parameter identification, the significant influences of flight altitude, image overlap and orientation of the camera axis relative to the object are to be mentioned, whereby the increased accuracy seems to be directly related to the stability of the image linkage or the number of valid point correspondences.

Keywords: Mine Surveying, UAV-Surveying, Photogrammetry, Structure from Motion, Accuracy investigation

\section{Einleitung}

Obwohl die technologischen Entwicklungen im Bereich geodätischer Sensoren und Verfahren in den letzten Jahrzehnten rasant fortgeschritten sind [1], hatte kaum eine technologische Neuentwicklung auf Anhieb einen derart starken Einfluss auf die markscheiderische und geodätische Praxis wie das Aufkommen unbemannter Fluggeräte (unmanned aerial vehicles, UAVs). Im Gegensatz zu vie- 
len herkömmlichen Messsystemträgern, wie Flugzeugen, Satelliten, Fahrzeugen oder Schiffen, zeichnen sich UAVs vor allem durch die Möglichkeit der schnellen, einfachen, sicheren und kostengünstigen Datenerfassung aus. Als Trägerplattform für verschiedene Sensorik erweitern sie den Raum, wo Vermessungen durchgeführt werden können, und schließen die (geometrische) Lücke zwischen klassischer Fernerkundung, Luftbildphotogrammetrie und terrestrischen Anwendungen [2,3].

\subsection{Motivation}

Auch in der bergmännischen Vermessung zählen unbemannte Flugsysteme, vor allem zur Nachtragung des Bergbaukartenwerks (\$42 [4]), zur Massenermittlung, sowie zur Erhebung des Ist-Zustandes bereits zum erweiterten Stand der Technik. Der Möglichkeit zur hochauflösenden und vor allem tagesaktuellen Herstellung von optisch realistischen 3D-Modellen aus Bildern kommt, insbesondere vor dem Hintergrund der fortschreitenden Digitalisierung und Automatisierung im Bergbau, besondere Bedeutung zu.

Die automatisierten Verfahren stellen jedoch hohe Anforderungen an die Qualität und vor allem an die geometrische Aufnahmekonfiguration der Bilder. Ist die Genauigkeit der klassischen Photogrammetrie hinlänglich untersucht, so gab es zu den neuen UAV-gestützten Anwendungen keine grundlegenden wissenschaftlichen Untersuchungen, welche Parameter in welchem Ausmaß für die erreichte Genauigkeit verantwortlich zeichnen. Im Gegensatz zur analogen und analytischen Photogrammetrie basiert SfM auf einer Vielzahl an automatischen und teilweise fehlerbehafteten Bildmessungen (Beobachtungen), was eine robuste Ausreißerelimination und nichtlineare Optimierung innerhalb der Auswertung notwendig macht. Entsprechend ist eine deterministische Fehlerbetrachtung im Sinne des Varianzfortpflanzungsgesetzes nur bedingt, bzw. gar nicht, möglich und quantitativ belastbare Aussagen zur erreichbaren Genauigkeit können auf diese Weise nicht wirklich gegeben werden [5].

Nichtsdestotrotz und auch obwohl der theoretische Unterbau oft nicht in ausreichendem Maße vorhanden ist, kommen UAVs in nahezu allen Bereichen der Geodatenerfassung immer häufiger zum Einsatz und werden dabei auch sehr oft von fachfremdem und nicht vermessungstechnisch geschultem Personal eingesetzt. Neben der gebotenen Sorgfalt zur Vermeidung grober und zufälliger Fehler müssen - ähnlich dem Nivellieren aus der Mitte oder dem Messen in zwei Kreislagen bei klassischen geodätischen Vermessungen - auch unbemannte Flugsysteme bzw. insbesondere die installierten Kameras "richtig“ verwendet werden, um die Auswirkung etwaig vorhandener systematischer Fehler zu vermeiden. Die "richtige Verwendung" zielt hier allerdings nicht nur auf die fotografische Güte der Bilder ab, sondern vielmehr auf die geometrische Qualität der dreidimensionalen Ergebnisse bzw. Punktwolken, die signifikant von der Erfahrung und den Fähigkeiten des Piloten beeinflusst wird. Vor diesem Hintergrund muss die Datenaufnahme in engem Zusam- menhang mit der nachgeschalteten Auswertung betrachtet werden, was insbesondere bei unerfahrenen und ungeschulten Anwendern nicht immer gegeben ist. Um eine definierte Genauigkeit und auch Vollständigkeit im Sinne einer markscheiderischen Ergebnisverantwortlichkeit zu gewährleisten, wäre es wahrscheinlich von Vorteil, den Faktor Mensch bzw. Anwender durch eine weitestgehend autonome Flugplanung zu eliminieren. Die theoretischen und mathematischen Grundlagen hierfür zu liefern war das Ziel der hier vorgestellten Dissertation und sollte vordergründig durch eine reproduzierbare Identifizierung und Quantifizierung der relevanten Einflussparameter erreicht werden.

\subsection{Untersuchungsgegenstand}

Wie bereits erwähnt, dienen unbemannte Fluggeräte heute als Trägerplattformen für verschiedenste Sensorik. Neben den für die Fluglageregelung notwendigen On-Board Sensoren existiert auch eine große Vielzahl an unterschiedlichen "'Sensing-Units'“, die von herkömmlichen RGB (Rot, Grün, Blau) Kameras über Multi- bzw. Hyperspektralkameras bis hin zu RADAR (Radio Detection and Ranging) und LiDAR (Light Detection and Ranging) Sensoren reichen [6-8].

Kern der gegenständlichen Forschung ist der Einsatz von Drohnen im Bergbau zur Erfüllung bzw. Ergänzung der markscheiderischen Aufgaben und Tätigkeiten. Dabei wird insbesondere auf die markscheiderischen Aufgaben im Tagbau abgestellt, die vom Anfertigen und Führen des Bergbaukartenwerks über die Beaufsichtigung der Vermessungen im Bergbau und Aufgaben der bergbaulichen Raumordnung und Sicherungspflicht bis hin zu bergschadenkundlichen Aufgaben, vor allem zum Schutz der Oberfläche und zur Sicherung der Oberflächennutzung nach Beendigung der Bergbautätigkeit, reichen [4, 9].

Unter Berücksichtigung der Verbreitung verfügbarer Sensoren beziehen sich die angestellten Untersuchungen auf "herkömmliche“ RGB Bilder unter Verwendung handelsüblicher Digitalkameras. Die Verwendung von Laserscanning Systemen in Kombination mit UAVs wird genauso wenig in die Betrachtung einbezogen wie etwaige Anwendungen mit Multi- oder Hyperspektralkameras.

Außerdem lag der Fokus auf der üblicherweise gewählten 3D-Rekonstruktion mittels automatisierter Mehrbildauswertung bzw. "'Structure from Motion (SfM)"“ [10]. $\mathrm{SfM}$ ist in der Lage, aus einem zunächst ungeordneten Set an Bildern die äußere Orientierung sowie eine punktbasierte Szenenrekonstruktion zu errechnen. Da die Aufnahmepositionen bei der UAV-Befliegung systembedingt unregelmäßig und nicht hochgenau bestimmbar sind, fußen nahezu alle Anwendungen auf dieser ursprünglich aus dem Bereich Computer Vision stammenden Algorithmik. Etwaige Einfluss- und Steuerparameter der Datenprozessierung und Bildauswertung wurden allerdings nicht in die Betrachtungen miteinbezogen. Die Auswertung mittels SfM erfolgte nach den immer gleichen Mustern und wurde in der Arbeit weitestgehend als "Black Box"“ betrachtet. Vielmehr lag der Fokus auf einer Analyse aus Sicht des markscheiderischen bzw. geodätischen Anwenders und 
zielte entsprechend auf die im Feld steuerbaren Einflussgrößen ab.

\subsection{Zielsetzung}

Bedingt durch den immer häufigeren Einsatz unbemannter Flugsysteme in den verschiedensten Bereichen der Vermessung gewinnt auch die Frage nach der tatsächlich erreichten Genauigkeit zusehends an Bedeutung. Die konkrete Zielsetzung der präsentierten Dissertation war daher eine robuste Identifizierung genauigkeitsrelevanter Einflussparameter bei der bildbasierten Vermessung unter Verwendung von Structure from Motion.

Auf Basis realmaßstäblicher, modellhafter und teilweise numerischer Überlegungen sollten die relevanten Parameter-genauigkeitswirksam, allerdings auch vom Anwender unmittelbar beeinflussbar - identifiziert, sowie deren Einfluss und insbesondere auch Wechselwirkung quantifiziert werden. Konkret sollte Antwort auf die folgenden Fragen gegeben werden [11]:

- Welche Parameter der Bildaufnahme beeinflussen die Genauigkeit der rekonstruierten 3D-Objekte bei der Auswertung mittels Structure from Motion?

- In welcher Form äußern sich die Einflüsse an den Ergebnissen? Kommt es zu systematischen Effekten bzw. Deformationen?

- Gibt es Gemeinsamkeiten bzw. Parallelen zur klassischen Photogrammetrie und wo liegen Unterschiede?

- Bestehen Wechselwirkungen bzw. Korrelationen zwischen den Einflussparametern? Und falls ja, in welcher Form?

- Wie muss die Datenaufnahme erfolgen, um reproduzierbare Ergebnisse mit ausreichender Genauigkeit zu gewährleisten?

- Welche Bedeutung kommt den Erkenntnissen bei der markscheiderischen Vermessung im Bergbau zu?

- Inwieweit ist die UAV-gestützte photogrammetrische Vermessung zur Erfüllung der markscheiderischen Aufgaben im Tagbau geeignet?

\section{Genauigkeit der UAV-Photogrammetrie}

Beim Studium vorhandener Literatur und vor allem auch thematisch relevanter Konferenzbeiträge zeigt sich deutlich, dass mit Kameras ausgerüstete UAVs eine wertvolle Ergänzung zu herkömmlichen Methoden darstellen können und dass sie durchaus in der Lage sind, einen Teil der markscheiderischen Aufgaben (hauptsächlich Nachtragsvermessung und Massenermittlung) unter Einhaltung der gesetzlichen Schranken zu übernehmen. Die erreichte 3DGenauigkeit liegt in der Größenordnung von rund 1,5 GSD, bzw. unter realen Einsatzbedingungen im Bereich weniger Zentimeter $(2,5$ bis $3 \mathrm{~cm})$ und somit in derselben Größenordnung wie bei RTK-GNSS. Daraus ergeben sich allerdings auch ganz klare Anwendungsgrenzen, sodass gesagt werden muss, dass z.B. Messungen zur Erfassung von Bodenbewegungen in der Größenordnung weniger Millimeter aktuell "noch“ nicht durchführbar sind.

Nahezu alle wissenschaftlichen Untersuchungen zeigen deutlich, dass ein unkontrolliertes und völlig freies Fotografieren trotz hoher Redundanz nicht zwingendermaßen zu einer hohen Objektgenauigkeit führt, und eine mit Sachverstand durchgeführte Aufnahmeplanung somit genauso wichtig ist wie die Auswahl einer geeigneten Kamera. Die besten Ergebnisse können üblicherweise durch eine Kombination verschiedener Aufnahmeentfernungen und Blickrichtungen erzielt werden: Nahe Aufnahmen liefern dabei den nötigen Detailgrad, aus größerer Entfernung aufgenommene Überblicksbilder stabilisieren den Bildverband und vermindern den durch die Fehlerfortpflanzung im Folgebildanschluss entstehenden Drift. Konvergente und verkantete Aufnahmen haben positive Auswirkung auf die Schnittgeometrie und die In-situ-Schätzung der radialen Verzeichnungsparameter, und die allgemein höhere Redundanz, die sich aus einer höheren Beobachtungsanzahl ergibt, führt zur numerischen Stabilisierung der nichtlinearen Optimierung. Überdies steht die erreichte Genauigkeit wie bei allen photogrammetrischen Verfahren - in unmittelbarem Zusammenhang mit der Bodenauflösung (Ground Sampling Distance, GSD) bzw. dem Aufnahmeabstand. Objekte, die kleiner sind als die Bildauflösung, können nicht erfasst werden, und entsprechend ist die erreichbare Genauigkeit in dieser Richtung klar begrenzt.

Bei näherer Analyse der möglichen genauigkeitsrelevanten Einflussparameter öffnet sich ein weites Feld. Neben dem offensichtlichen Aufnahmesystem bzw. der Kamera müssen vor allem die Aufnahmekonfiguration (Parameter der Bildaufnahme) sowie die Art der Bildauswertung und der Maßstabs- und Datumsfestlegung genannt werden. Natürlich ist hier auch noch die Beschaffenheit des aufzunehmenden Objekts zu nennen, wobei dies selten vom Anwender beeinflusst werden kann und die einzige Eingriffsmöglichkeit in der Regel durch den Verzicht oder die zeitliche Verschiebung des Messeinsatzes gegeben ist.

Es existiert somit eine Reihe unterschiedlicher Einflussgrößen und abhängig vom Anwendungsfall bzw. Autor steht eine andere im Zentrum der Betrachtungen. So stellen z. B. [12] oder [13] sehr stark auf die eingesetzte Kamera $a b$, während bei [14] oder [15] die Frage der Georeferenzierung im Zentrum steht. Die präsentierten Ergebnisse bzw. vor allem auch die erkannten systematischen Fehlermuster deuten allerdings klar darauf hin, dass - viel mehr als in der klassischen Photogrammetrie mit kalibrierten Messkameras - starke Korrelationen zwischen den verschiedenen Einflussparametern bestehen, weshalb alle Parameter gemeinsam und auf keinen Fall nur isoliert betrachtet werden dürfen. Entsprechend schwierig ist es auch, erkannte Phänomene bzw. systematische Effekte an einer bestimmten Ursache festzumachen, oder aber allgemein gültige Empfehlungen aus der Analyse eines einzelnen Parameters zu geben. Die Aufnahmekonfiguration beispielsweise soll mehrere Aufgaben erfüllen: Offensichtlich soll zunächst einmal versucht werden, das gesamte Objekt mit Bildern abzudecken, um Vollständigkeit sowie den ausreichenden Detailgrad zu gewährleisten. Zusätzlich soll die Konfiguration aber ausreichend stabil sein, um eine 


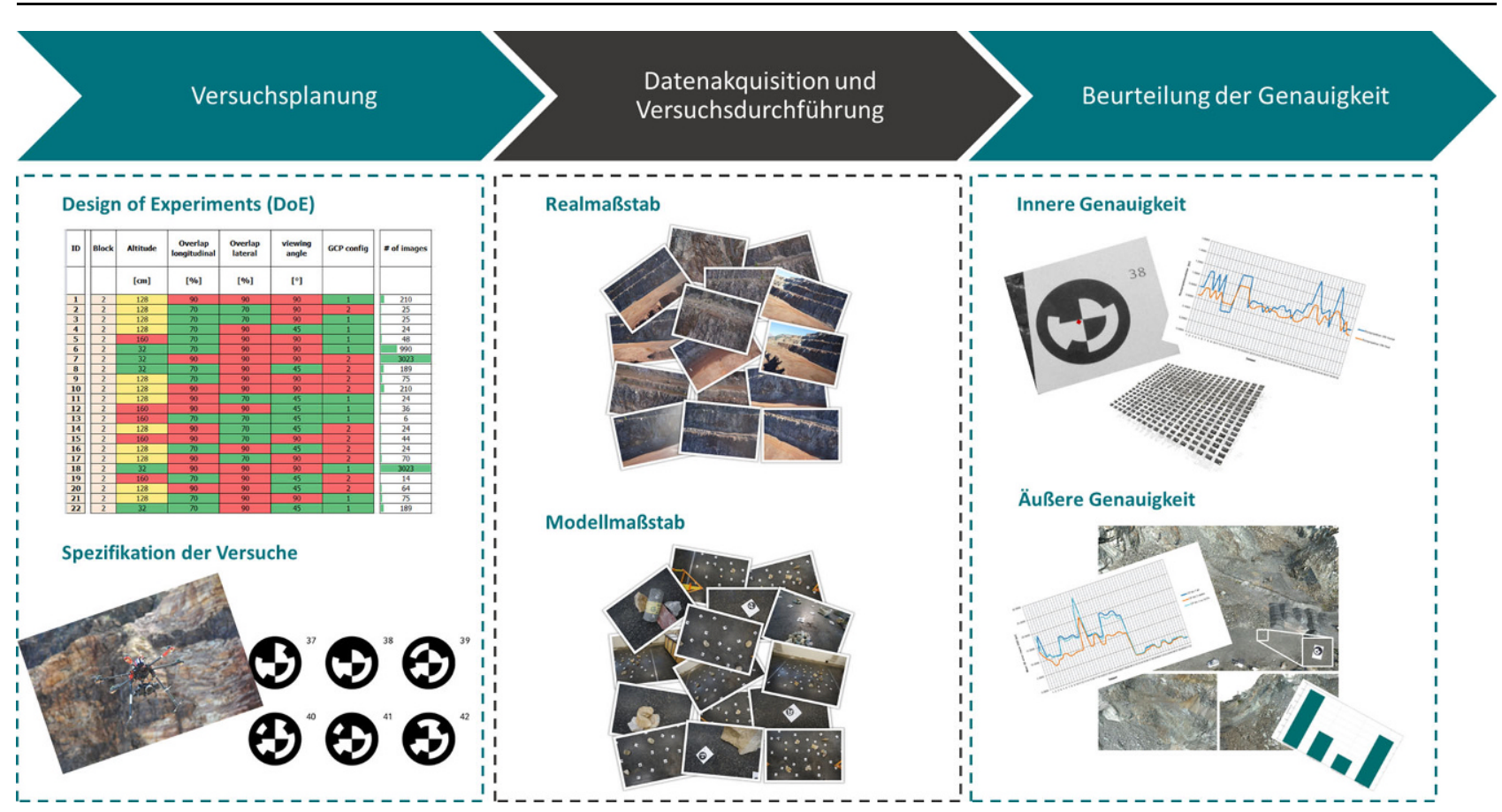

Abb. 1: Die angewandte Forschungsmethodik umfasst im Wesentlichen 3 Abschnitte: die Versuchsplanung nach dem Konzept der Statistischen Versuchsplanung, die Versuchsdurchführung in Form von Bildaufnahmen im Real- und Modellmaßstab, und die Beurteilung der erreichten Genauigkeit wobei sowohl innere als auch äußere Genauigkeitsmaßstäbe angelegt werden [11]

physikalisch richtige Schätzung der inneren Orientierung zu ermöglichen, und gleichzeitig soll sowohl die Zeit für die Bildaufnahme als auch die angehäufte Datenmenge möglichst gering gehalten werden.

Ein typisches "Fehlerbild“ der SfM-Auswertung ist der "Dome Effekt" [16-18]. Das heißt, der gesamte Bildverband neigt zu einer kuppelförmigen Verkrümmung, deren Ursache in der klassischen Photogrammetrie zumeist an einer fehlerhaft geschätzten inneren Orientierung (Kamerakonstante und Radialverzeichnung) festgemacht wird. Bei Structure from Motion allerdings handelt es sich hier sehr häufig um "künstlich fehlerhafte" intrinsische Parameter, die sich als numerisches Optimum in der Bündelblockausgleichung ergeben, wenn der Bildverband oder die Passpunktkonfiguration für eine derartige Optimierung nicht geeignet sind. Anders ausgedrückt sind charakteristische "domartige“ Fehlermuster zumeist ein Ergebnis fehlerhafter intrinsischer Parameter. Die Fehler in der inneren Orientierung ergeben sich allerdings oftmals als Folge externer Parameter wie z. B. einer ungeeigneten Aufnahmeanordnung, die aufgrund der stark automatisierten Auswertung und der auf inneren Genauigkeitsmaßen basierenden Optimierung nahezu ungefiltert Eingang in die Ergebnisse findet.

\section{Methodik}

Zur Beantwortung der in Abschn. 1.3 aufgeworfenen Fragen bedarf es einer tiefgreifenden wissenschaftlichen Untersuchung. Das Ziel ist die Beschreibung und Untersuchung eines komplexen multivariaten Systems mit verschiedenen
Eingangs- und Ergebnisgrößen, wobei der Fokus auf der robusten Identifikation genauigkeitswirksamer Steuerparameter sowie der Ableitung belastbarer Relationen liegt.

Aus den dargestellten Überlegungen und den daraus abgeleiteten Zielen ergibt sich die in Abb. 1 ersichtliche Herangehensweise bzw. auch der Ablauf der durchgeführten Untersuchungen. Im Wesentlichen umfassen die praktischen Versuche drei Abschnitte: die Versuchsplanung, die Versuchsdurchführung und Datenakquisition und die Analyse der aufgenommenen Daten bzw. die Beurteilung der erreichten Genauigkeiten.

Vor allem bei der Untersuchung eines komplexen Systems mit mehreren Eingangs- und Ausgangsgrößen kommt der Versuchsplanung besondere Bedeutung zu. Insbesondere in der industriellen bzw. ingenieurwissenschaftlichen Anwendung (Produktentwicklung, Prozessoptimierung) stellt die Statistische Versuchsplanung (Design of Experiments, DoE) als Methodik zur systematischen Planung und statistischen Auswertung von Versuchen ein wichtiges Werkzeug dar. Mit verhältnismäßig geringem Aufwand wird durch strukturierte Planung der funktionale Zusammenhang von Einflussparametern und Ergebnissen ermittelt bzw. mathematisch beschrieben. Aus den Ergebnissen kann erkannt werden, welche Werte der Steuergrößen besonders günstig für verschiedene Zielgrößen sind, aber vor allem auch, welche Relationen zwischen den einzelnen Steuergrößen bestehen und welche Kompromisse gegebenenfalls eingegangen werden müssen [19-21].

Unter Berücksichtigung einer großen Anzahl an Vorversuchen [2], der praktischen und betrieblichen Einschränkungen sowie der in Abschn. 2 präsentierten Literaturanalyse 
wurden die folgenden 5 Faktoren bzw. Faktorstufen festgelegt, die überdies auch in den meisten verfügbaren Flugplanungswerkzeugen implementiert sind:

- Flughöhe (A): getestet auf 2 bzw. 3 Stufen, 30 und 150 m im Realmaßstab, 32, 128 und $160 \mathrm{~cm}$ im Modellmaßstab 1:100

- Überlapp longitudinal (B): getestet auf 2 Stufen, 70 und $90 \%$

- Überlapp lateral (C): getestet auf 2 Stufen, 70 und $90 \%$

- Blickwinkel relativ zum Objekt (D): getestet auf 2 Stufen, 45 und $90^{\circ}$

- Passpunktkonfiguration zur Georegistrierung (E): 2 verschiedene räumliche Konfigurationen mit je 6 Passpunkten getestet

Versuchsdurchführung ist im vorliegenden Fall gleichbedeutend mit Bildaufnahme bzw. Befliegung, wobei die Datenakquisition sowohl unter realen Bedingungen in ausgewählten Bergbauen als auch im Modellmaßstab und unter kontrollierten Witterungs- und Belichtungsverhältnissen durchgeführt wurde.

Die Datenanalyse bzw. die Beurteilung der Ergebnisse erfolgte in mehrfacher Form bzw. aus verschiedenen Blickwinkeln: Es wurde eine Unterscheidung in innere und äußere Genauigkeit vorgenommen, wohl wissend, dass diese beiden Begriffe in der herkömmlichen Geodäsie in leicht anderem Zusammenhang üblich sind. Die innere Genauigkeit umfasst dabei jene Ergebnismerkmale, die sich aus der alleinigen Auswertung der Bilder ergeben und somit keine unmittelbare metrische Qualität aufweisen. Die Beurteilung erfolgt im Bild- bzw. maximal im Modellkoordinatensystem und umfasst konkret die Ergebnisse der Simultankalibrierung, die verbliebenen Rückprojektionsfehler nach der Bündelblockausgleichung und die Stabilität der Bildverknüpfung in Form der Anzahl gültiger Epipolargeometrien zwischen den Stereopartnern. Die äußere Genauigkeit hingegen zielt auf den Vergleich der photogrammetrisch erzeugten 3D-Modelle mit übergeordnet genauen Referenzmodellen ab und schlägt somit die Brücke zur praktischen Anwendung und metrischen Genauigkeitsbeurteilung.

\section{Untersuchungen}

Nachdem die zu untersuchenden Parameter und auch der Ablauf der Versuche festgelegt wurden, sollten diese in Form von realer Bildaufnahme bzw. UAV-Befliegung durchgeführt werden, wobei besonders darauf geachtet wurde, alle übrigen Einflussgrößen möglichst konstant zu halten. Alle im Rahmen der vorliegenden Dissertation präsentierten Untersuchungen basieren somit auf realen Bildern und „echten“ Kameras. Simulationsbasierte Ansätze (z. B. [22]) mit „virtuellen“ Bildern und Kameramodellen werden entsprechend nicht angewandt bzw. näher behandelt.

\subsection{Versuchsanordnung}

Um die Beurteilung der Genauigkeit zu ermöglichen, wurden zunächst geeignete Testobjekte ausgewählt und vorbereitet sowie Evaluierungsmöglichkeiten geschaffen, wobei eine objektive Analyse nur über ein mit übergeordneter Genauigkeit hergestelltes Referenzmodell möglich ist. Da es sich bei der Photogrammetrie um ein flächiges Messverfahren handelt, das die Objektoberfläche in hohem Detailgrad in Form einer 3D-Punktwolke wiedergibt, wäre auch beim Referenzmodell eine Punktwolke eine geeignete Möglichkeit. Diese könnte zum Beispiel mit terrestrischem Laserscan in ausreichender Genauigkeit erzeugt werden, und die auftretenden Differenzen könnten flächig visualisiert und analysiert werden. Eine weitere Möglichkeit wäre ein dichtes Netz an sowohl in den Bildern als auch in der Örtlichkeit eindeutig identifizierbaren Referenzpunkten, was auch im gegenständlichen Fall zur Anwendung kam. Signalisierte Pass- und Kontrollpunkte können sowohl mit herkömmlichen Vermessungsmethoden im Feld (z. B. Tachymetrie) als auch durch Bildmessung eindeutig erkannt werden, was eine eindeutige Gegenüberstellung identer Objektpunkte ermöglicht und die Evaluierung von systematischen Einflüssen der Referenzvermessung befreit. Da die Bildmessgenauigkeit für signalisierte Punkte in einer ähnlichen Größenordnung liegt wie für Natural Features, ist davon auszugehen, dass die Genauigkeit der speziell signalisierten Punkte auf jeden einzelnen Verknüpfungspunkt und somit auf die Punktwolke übertragbar ist.

\subsection{Versuchsdurchführung}

Wie bereits erwähnt, erfolgte die Bildaufnahme unter Realbedingungen und zusätzlich auch im Modellmaßstab. Einerseits kann es durchaus als gültige wissenschaftliche Praxis betrachtet werden, grundsätzliche Zusammenhänge zunächst am Modell zu erforschen und anschließend die Gültigkeit der getroffenen Aussagen in der Realität zu verifizieren [23, 24]. Andererseits bieten modellhafte Versuche oftmals die Möglichkeit, Faktorstellungen mit höherer Genauigkeit zu steuern, bzw. sind auch Faktorkombinationen, die in der Natur nur schwer realisierbar sind, zumeist einfach herzustellen. Außerdem können im Labor die Umgebungsbedingungen weitestgehend konstant gehalten werden, und nicht kontrollierbare Störeinflüsse (z. B. Wind) werden minimiert. Da die photogrammetrisch erzeugten Modelle zunächst maßstabsfrei und geometrisch ähnlich sind (nur aus den Bildern ist die metrische Größe der aufgenommenen Objekte nicht definiert), werden die Versuchsergebnisse durch die Skalierung auch in keiner Weise verfälscht und die Versuche sollten unabhängig vom gewählten Maßstab zu vergleichbaren Ergebnissen führen.

Bereits aus den gewählten Faktoren und den zugehörigen Stufen geht hervor, dass die Bildaufnahme, wie bei den meisten UAV-Einsätzen üblich, in regelmäßigem Raster und parallelen Bahnen in einer definierten Höhe über dem Gebiet erfolgte. Um die statistische Aussagekraft der Ergebnisse zu erhöhen und auch Aussagen zur Reproduzierbarkeit und Belastbarkeit der Ergebnisse treffen zu kön- 
Abb. 2: Wird der mittlere 3D-Punktfehler durch die theoretische mittlere GSD dividiert, kann ein eindeutiger Einfluss derFlughöhe erkannt werden, wobei die hohen Flüge zu höheren Genauigkeiten führen (Datensätze sind nach aufsteigender Flughöhe sortiert). Nach [11]

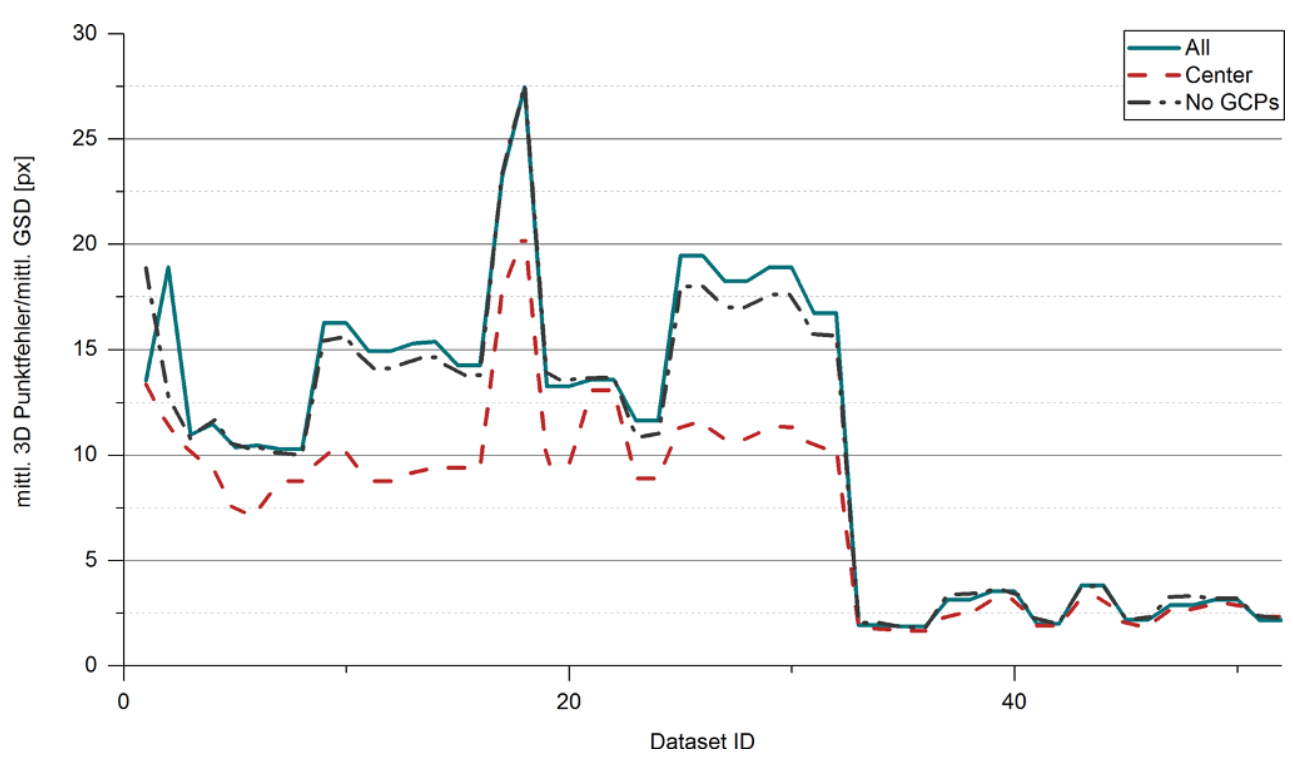

nen, wurde außerdem jeder Bildverband zweimal unter den exakt selben Bedingungen getestet. Ebenso wurden alle Aufnahmen unter Verwendung desselben Kameramodells durchgeführt. Auf diese Weise konnten Einflüsse des Aufnahmesystems zwar nicht gänzlich ausgeschlossen, aber zumindest konstant gehalten werden.

Die Auswertung erfolgte unter Verwendung der SfM-Pipeline des Instituts für maschinelles Sehen und Darstellen (ICG) an der TU Graz (TUG) in der Windows Version vom 08.04.2016. Der wesentliche Vorteil gegenüber kommerziellen Softwareprodukten wie z. B. Agisoft Metashape [25] besteht in der Zugänglichkeit zum Source Code und somit in der eindeutigen Festlegung der Auswerteparameter. Auf diese Weise konnten vorhandene Einflüsse der Auswerteroutine weitestgehend ausgeschaltet und die angestrebten Analysen zielgerichtet durchgeführt werden.

\section{Ergebnisse}

Wie bereits erwähnt, erfolgte die Beurteilung der Genauigkeit nach äußeren und inneren Genauigkeitsmaßen. Während bei der äußeren Genauigkeit für jeden Versuchsdurchlauf bzw. Bildverband die Koordinaten der Kontrollpunkte bestimmt und diese den bekannten Referenzkoordinaten aus der herkömmlichen Vermessung gegenübergestellt wurden, geben die Größen der inneren Genauigkeit Hinweise auf etwaige Begründungen und Ursachen für die zu Tage getretenen Phänomene, was neben der reinen Beschreibung der erkannten Effekte eines der wesentlichsten Ziele der vorliegenden Forschung ist.

Da in vielen Fällen üblich und zur besseren Vergleichbarkeit der Ergebnisse im Real- und Modellmaßstab, wurden die mittleren Fehler für die folgenden Darstellungen auch durch die mittlere GSD dividiert und die Fehler somit als Vielfaches der theoretischen Bodenauflösung dargestellt.

\section{1 Äußere Genauigkeit}

Werden alle Datensätze zusammengefasst betrachtet, so zeigt sich zunächst einmal, dass die erreichte mittlere Genauigkeit zwischen 1,9 und 27,5 GSD liegt, wobei eine markante Sprungstelle mit der Flughöhe erkennbar ist. Entgegen der ursprünglichen Erwartung, dass eine höhere Auflösung bzw. geringere Flughöhe zu einer höheren Genauigkeit führt, zeigt sich anhand der durchgeführten Flüge genau das Gegenteil. Die „150m Flüge“ sind auffallend genauer als jene, die in $30 \mathrm{~m}$ Höhe aufgenommen wurden, was auch in Abb. 2 deutlich erkannt werden kann, wo die Datensätze nach aufsteigender Flughöhe geordnet dargestellt sind.

Wird der Verlauf der Kurven in Abb. 2 noch stärker hinsichtlich Korrelation mit den Faktorstufen analysiert, so zeigt sich, dass Schrägsicht zu höheren Genauigkeiten führen kann als Nadiraufnahmen, und dass ein höherer Überlapp naturgemäß die Genauigkeit erhöht, wobei der laterale Überlapp einen stärkeren Einfluss aufweist als jener in longitudinaler Richtung. In Abb. 2 kann dies an den „Höckern“ im Linienverlauf erkannt werden, wobei die Schwankungen bei geringer Flughöhe (Dataset ID 1-32) deutlicher zu erkennen sind als bei den Aufnahmen aus großer Höhe, was allerdings vordergründig auf die größeren Fehlerbeträge zurückzuführen ist.

Deutlicher werden die erwähnten Effekte anhand der Box-Plot Diagramme in Abb. 3, wo bei a der Einfluss der Flughöhe, bei $b$ jener des Blickwinkels, bei $c$ und d der Einfluss der Bildüberlappung, bei e jener der Passpunktanordnung und bei $f$ der Einfluss der Versuchswiederholung dargestellt ist.

\subsection{Innere Genauigkeit}

Obwohl bei nahezu allen untersuchten Größen der inneren Genauigkeit ein Zusammenhang mit den Parametern der Bildaufnahme erkannt werden konnte, soll an dieser Stelle 

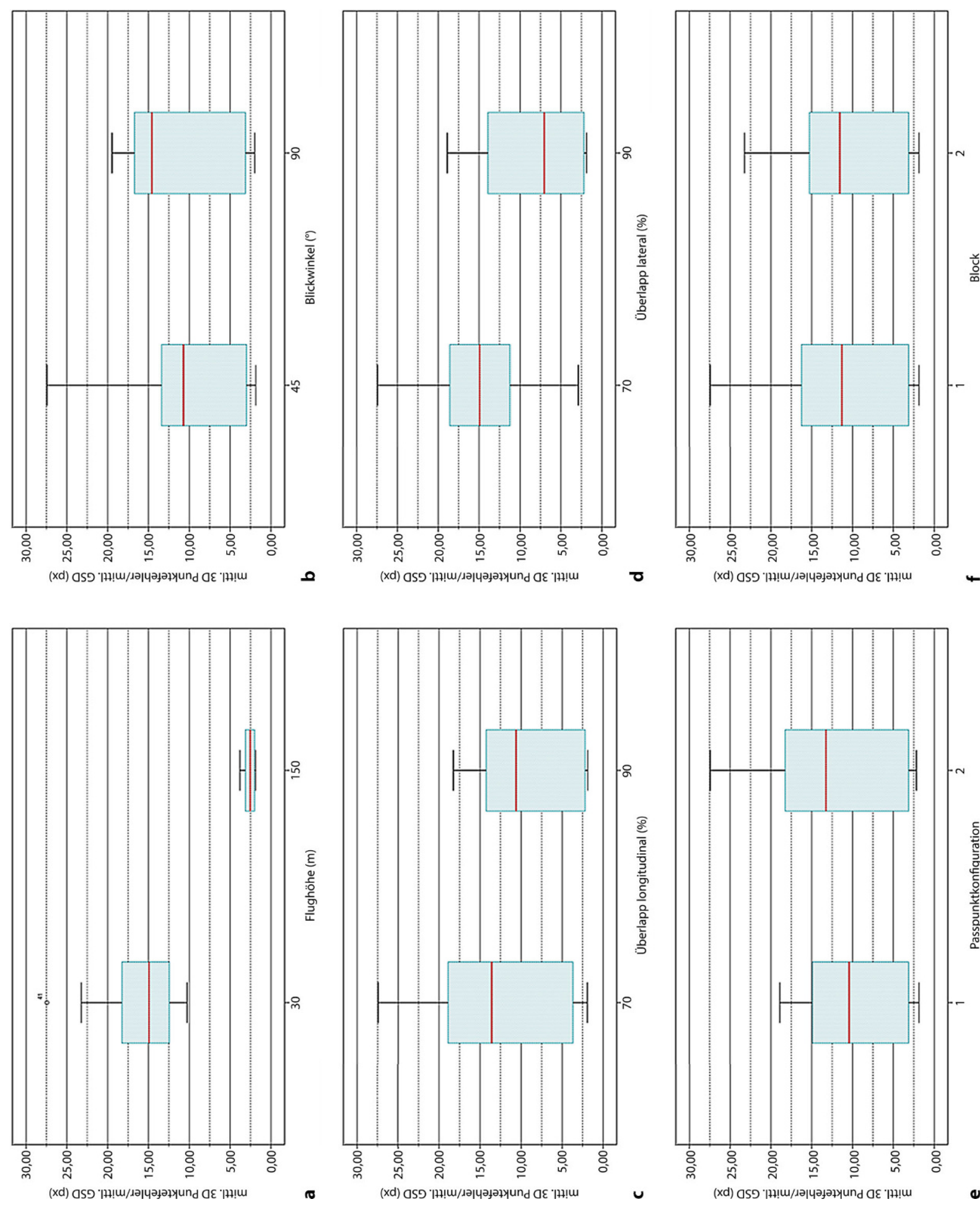

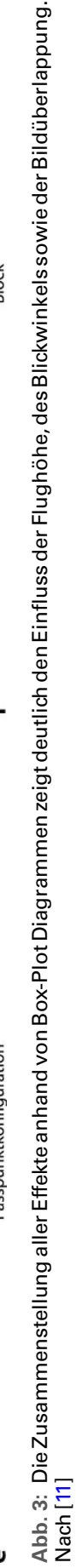


Abb. 4: Die mittl. Verknüpfungsanzahl zwischen den Bildern reproduziert sehr deutlich die äußere Genauigkeit in Abb. 2 und gibt ein nahezu gespiegeltes Bild [11]

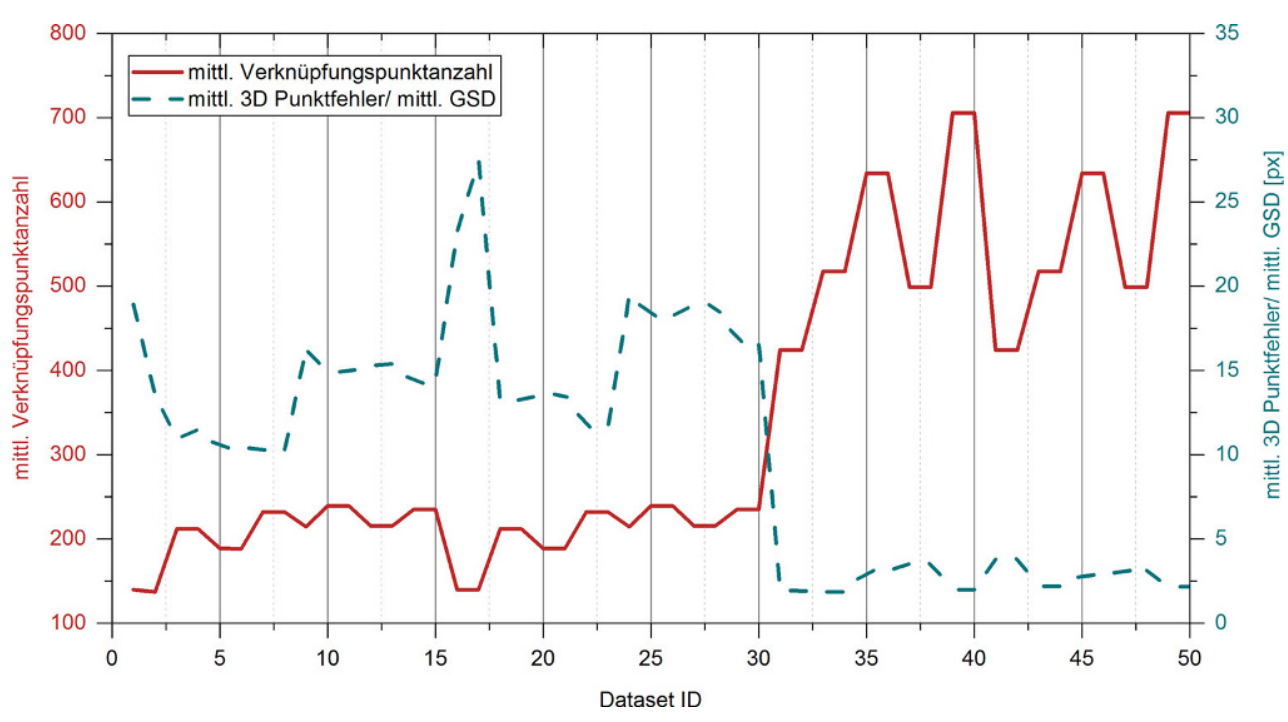

\section{Schlussfolgerungen und Ausblick}

die Anzahl der gültigen Verknüpfungen zwischen den Bildern besonders hervorgehoben werden. Je höher die Anzahl an gefundenen und gültigen Punktkorrespondenzen, desto stärker überbestimmt ist die Schätzung der relativen Orientierung der Bilder zueinander, und zumindest rein mathematisch müsste dies auch zu höherer Genauigkeit im Ergebnis führen.

Wird die entsprechende Kurve in Abb. 4 (die Ordnung der Datensätze ist ident mit jener in Abb. 2) betrachtet, kann ein deutlicher systematischer Zusammenhang erkannt werden. Auch hier ist die sprunghafte Veränderung mit der Flughöhe deutlich zu erkennen. Bei größerer Flughöhe existiert eine größere Anzahl an gültigen Verknüpfungen zwischen den Bildern als bei geringerer Aufnahmedistanz, was zwar zunächst durch den größeren Bildausschnitt logisch erscheint, allerdings dürfte dieser Umstand aufgrund des konstanten und prozentuell ermittelten Überlapps in beiden Höhen nicht von Bedeutung sein.

Noch deutlicher wird die angesprochene Systematik, wenn die äußere Genauigkeit mit der mittleren Anzahl an gültigen Verknüpfungen in Zusammenhang gebracht wird. (siehe Abb. 4). Die rote Kurve, die die mittlere Verknüpfungsanzahl zeigt, verläuft nahezu gespiegelt zu den Ergebnissen der äußeren Genauigkeit, die in diesem Fall wieder als Vielfaches der GSD dargestellt ist. Es ist zwar zu erwarten, dass die Aufnahmeanordnung Auswirkung auf die Anzahl der Verknüpfungen zwischen den Bildern und damit auf die Stabilität des Bildverbands hat, dass diese Anzahl aber in unmittelbarem Zusammenhang mit der erreichten äußeren Genauigkeit steht, war so eindeutig nicht vorhersehbar.

Die mittlere Anzahl an gültigen Bildverknüpfungen erscheint klar geeignet, um Rückschlüsse auf die erreichte äußere Genauigkeit zu ziehen, und außerdem legen die Ergebnisse nahe, dass die äußere Genauigkeit vordergründig durch die Verknüpfungspunktanzahl beeinflusst wird, die wiederum starke Abhängigkeit von den geometrischen Parametern der Aufnahmeanordnung zeigt.
Die Ergebnisse der hier präsentierten Dissertation können aus verschiedenen Blickrichtungen betrachtet werden und sind in ihrer Bedeutung entsprechend vielfältig. Zunächst konnte gezeigt werden, dass bei "richtigem" Einsatz (Systemdesign, Flugplanung und Auswertung) die Genauigkeitsanforderungen der Markscheideverordnung [26] eingehalten werden können. Die UAV-gestützte Vermessung kann somit im Rahmen der Systemgrenzen - so können z. B. Absteckungsarbeiten nicht durchgeführt werden - eine wertvolle Ergänzung zur Erfüllung der markscheiderischen Aufgaben im Tagbau sein und wird als solche bereits zunehmend eingesetzt. Aus wissenschaftlich-theoretischer Sicht konnte mittels tiefgreifender Analyse praktischer Daten im Real- und Modellmaßstab eine statistisch signifikante Auswahl genauigkeitsrelevanter Einflussparameter gefunden werden. Außerdem erlaubte die Untersuchung verschiedener "Ausprägungen“ der Ergebnisse - äußere und innere Genauigkeit - vor allem auch Begründungen für die erkannten Phänomene zu geben.

Der deutlichste Zusammenhang konnte zwischen der erreichten äußeren Genauigkeit und der Anzahl an gültigen Verknüpfungen zwischen den Bildern erkannt werden. Je stärker die Verknüpfung zwischen den Bildern, bzw. je größer die Anzahl an gültigen Verknüpfungspunkten, desto höher ist auch die äußere Genauigkeit der rekonstruierten Objektpunkte. Zumindest zum relativen Vergleich zwischen Datensätzen erscheint diese Größe als belastbares Maß der Genauigkeitsbeurteilung und zeigt vor allem einen wesentlich stärkeren Zusammenhang mit den erreichten äußeren Genauigkeiten als beispielsweise die mittleren Rückprojektionsfehler. In praktischer Hinsicht kann dieser Zusammenhang vor allem dazu genutzt werden, um Bilder ohne externen Vergleichsmaßstab aus Gründen der Genauigkeit von der Auswertung auszuschließen, bzw. kann dieses Kriterium als robuste Fehlerfunktion der äußeren Genauigkeit in die Optimierung bzw. die Bündelblockausgleichung integriert werden. 
Einer der markantesten Unterschiede zur klassischen Photogrammetrie besteht darin, dass die Flughöhe einen unerwarteten Einfluss zeigt. Die reine Erhöhung der Bildauflösung scheint auf automatische Feature Detektoren und vor allem auch das Feature Matching einen anderen Einfluss zu haben als bislang angenommen. Somit kann gesagt werden, dass die geometrische Aufnahmeanordnung einen anderweitig ausgeprägten Einfluss auf die Auswertung hat, als dies bei klassisch photogrammetrischen Ansätzen der Fall ist. Für klassisch photogrammetrische Auswertung ideal geeignete Bildverbände treffen die Anforderungen von SfM nicht im Kern, und entsprechend sind auch die Ergebnisse zum Teil negativ überraschend. Die einzelnen Verfahrensschritte von SfM dürfen nicht isoliert betrachtet werden, sondern die Interaktionen zwischen den Prozessschritten müssen miteinbezogen werden.

\subsection{Handlungsempfehlung}

Neben der wissenschaftlichen Befassung und Analyse soll allerdings auch eine Handlungsempfehlung und Unterstützung für UAV-Anwender gegeben werden. Kurz und prägnant sollen im Folgenden die wichtigsten „Regeln“ für den Piloten im Feld zusammengefasst werden, wobei für die theoretische und experimentelle Begründung zu den getätigten Aussagen auf die dem Beitrag zu Grunde liegende Dissertation verwiesen wird [11]. Der Unterschied zu etwaig bereits vorhandenem „Erfahrungswissen“ besteht somit in der wissenschaftlichen Grundlage der getroffenen Empfehlungen, was diesen stärkeres Gewicht verleiht.

1. Bildaufnahme aus großen Flughöhen zeigt positiven Einfluss auf die erreichte äußere Genauigkeit

2. Starke Bildüberlappung in beiden Richtungen stabilisiert den Bildverband

3. Geneigte Orientierung der Aufnahmeachse wirkt stabilisierend

4. „Structure from Motion braucht Motion“, bzw. eine ausreichende Kamerabewegung und "Unterschiedlichkeit" zwischen den Bildern und Aufnahmepositionen

5. Für vermessungstechnische Genauigkeit sind Passpunkte erforderlich

6. Kombination verschiedener Faktorstellungen (insbesondere Blickwinkel) steigert die Genauigkeit

Einschränkend muss jedoch angemerkt werden, dass auch an dieser Stelle die Grenzen des definierten Untersuchungsgegenstands aufrecht bleiben, die vorgebrachten Empfehlungen sich somit auf die vom Piloten im Feld beeinflussbaren Größen beschränken und beispielsweise das Aufnahmesystem als solches nicht betrachtet wird.

\subsection{Ausblick}

Wird der Blick in die Zukunft gewagt, bilden die Erkenntnisse der präsentierten Dissertation zunächst einmal die notwendige Basis, um das Anwendungsfeld für UAVs weiter zu vergrößern. Das bessere Verständnis der erreichbaren
Genauigkeiten und im Besonderen auch das tiefere Wissen über deren Zustandekommen erlauben einen Blick über den aktuellen Tellerrand und eröffnen neue - bisher noch nicht erreichbare - Möglichkeiten. Im markscheiderischen Umfeld ist hier mit Sicherheit die Beobachtung von geogenen und anthropogenen Veränderungen zu nennen, wo die flächige Analyse einerseits zur Erhöhung der Sicherheit und andererseits auch zu einem besseren Verständnis der Versagensmechanismen beitragen könnte. Vor allem für hochgenaue Beobachtungsmessungen müssen die eingesetzten Instrumente "wirklich beherrscht" werden und alle möglichen Störeinflüsse schon in der Planung berücksichtigt werden.

Funding. Open access funding provided by Montanuniversität Leoben.

Open Access Dieser Artikel wird unter der Creative Commons Namensnennung 4.0 International Lizenz veröffentlicht, welche die Nutzung, Vervielfältigung, Bearbeitung, Verbreitung und Wiedergabe in jeglichem Medium und Format erlaubt, sofern Sie den/die ursprünglichen Autor(en) und die Quelle ordnungsgemäß nennen, einen Link zur Creative Commons Lizenz beifügen und angeben, ob Änderungen vorgenommen wurden.

Die in diesem Artikel enthaltenen Bilder und sonstiges Drittmaterial unterliegen ebenfalls der genannten Creative Commons Lizenz, sofern sich aus der Abbildungslegende nichts anderes ergibt. Sofern das betreffende Material nicht unter der genannten Creative Commons Lizenz steht und die betreffende Handlung nicht nach gesetzlichen Vorschriften erlaubt ist, ist für die oben aufgeführten Weiterverwendungen des Materials die Einwilligung des jeweiligen Rechteinhabers einzuholen.

Weitere Details zur Lizenz entnehmen Sie bitte der Lizenzinformation auf http://creativecommons.org/licenses/by/4.0/deed.de.

\section{Literatur}

1. Tscharf, A.: Technische Entwicklungen und Möglichkeiten im Markscheidewesen. BHM 165 (2020), H. 2, S. 111-116

2. Tscharf, A.: Potentiale und Herausforderungen neuer Vermessungstechnologien im Bergbau - Der Einsatz unbemannter Flugkörper zur Erfassung von Geodaten. BHM 161 (2016), H. 10, S. 481-487

3. Rumpler, M.; Hoppe, C.; Wendel, A.; Mayer, G.; Bischof, H.: EchtzeitQualitätsüberprüfung für zuverlässige UAV-gestützte Bilddatenerfassung und exakte, automatisierte Mehrbildauswertung, VGI Österreichische Zeitschrift für Vermessung und Geoinformation 101 (2013), Nr. 2+3, S. 88-100

4. Bundesgesetz über mineralische Rohstoffe (Mineralrohstoffgesetz MinroG), BGBI. I Nr. 38/1999

5. Rumpler, M.; Daftry, S.; Tscharf, A.; Prettenthaler, R.; Hoppe, C. Mayer, G.; Bischof, H.: Automated End-to-End Workflow for Precise and Geo-accurate Reconstructions Using Fiducial Markers. In Photogrammetric Computer Vision - PCV 2014, Proceedings, pp. 135-142

6. Briese, C.; Fortner, R.; Sager, P.; Pfeifer, N.: Vom Modellflughobby zu unbemannten Flugsystemen für die Geodatenerfassung, VGI Österreichische Zeitschrift für Vermessung und Geoinformation 101 (2013), Nr. 2+3, S. 64-74

7. Gaisecker, T.; Riegl, U.; Amon, P.; Pfennigbauer, M.: RIEGL LiDARLösungen für die UAV-basierte Vermessung. In: Unmanned Aeria Vehicles 2017 (UAV 2017). Ed. by DVW - Gesellschaft für Geodäsie, Geoinformation und Landmanagement e. V. Augsburg: Wißner-Verlag, 2017, ISBN: 978-3-95786-107-8. (Schriftenreihe des DVW, 86)

8. Genschow, D.: Anwendung von Radarsensoren in UAV. In: Unmanned Aerial Vehicles 2017 (UAV 2017). Hrsg.: DVW - Gesellschaft für Geodäsie, Geoinformation und Landmanagement e.V. Augsburg: Wißner-Verlag, 2017, ISBN: 978-3-95786-107-8. (Schriftenreihe des DVW, 86) 
9. Tscharf, A.: Stand der Technik in der Tagbauvermessung. BHM 163 (2018), H. 2, S. 57-63

10. Hartley, R.; Zisserman, A.: Multiple View Geometry in Computer Vision, 2.ed., Cambridge University Press, 2003

11. Tscharf, A.: UAV-gestützte Vermessung im Bergbau - Zur Frage der Genauigkeit unter Verwendung von Structure from Motion, Diss., Leoben, Montanuniv., Lehrst. F. Bergbaukunde, Bergtechnik und Bergwirtschaft, 2020

12. Greiwe, A.; Peters, J.; Spreckels, V.; Schlienkamp, A.; Lösler, M.; Eschelbach, C.: Geeignete Kameras zur Geländeaufnahme durch UAS. In: Luhmann, T.; Schumacher, C. (Hrsg.): Photogrammetrie, Laserscanning, Optische 3D-Messtechnik. Berlin, Offenbach: Wichmann, 2017, S. 202-221. ISBN: 3879076251

13. Cramer, M.; Leinss, B.: Welche ist am besten? - Anmerkungen zur Auswahl von Kamerasystemen in der UAS-Luftbildphotogrammetrie. In: UAV 2016 - Vermessung mit unbemannten Flugsystemen. Hrsg.: DVW - Gesellschaft für Geodäsie, Geoinformation und Landmanagement e.V. Augsburg: Wißner-Verlag, 2016, S. 97-118, ISBN: 978-3-95786-067-5 (Schriftenreihe des DVW, 82)

14. Przybilla, H-J.; Reuber, C.; Bäumker, M.; Gerke, M.: Untersuchungen zur Genauigkeitssteigerung von UAV-Bildflügen. In: Kersten, T. P. (Hrsg.): Bridging Scales - Skalenübergreifende Nah- und Fernerkundungsmethoden, Proceedings Wissenschaftlich-Technische Jahrestagung der DGPF in Köln, Hamburg: DGPF, 2015

15. Sanz-Ablanedo, E.; Chandler, J.; Rodríguez-Pérez, J.; Ordóñez, C.: Accuracy of Unmanned Aerial Vehicle (UAV) and SfM Photogrammetry Survey as a Function of the Number and Location of Ground Control Points Used. Remote Sensing 10 (2018), iss.10, p. 1606, ISSN: 2072-4292, https://doi.org/10.3390/rs10101606

16. Fraser, C. S: Automatic Camera Calibration in Close Range Photogrammetry. Photogrammetric Engineering \& Remote Sensing 79 (2013), iss.4, pp. 381-388. ISSN: 00991112, doi:10.14358/PERS.79.4.381

17. James, M. R.; Robson, S.: Mitigating systematic error in topographic models derived from UAV and ground-based image networks. Earth Surface Processes and Landforms 39 (2014), iss.10, pp. 1413-1420. ISSN: 01979337, https://doi.org/10.1002/esp.3609

18. Javernick, L.; Brasington, J.; Caruso, B.: Modeling the topography of shallow braided rivers using Structure-from-Motion photogram- metry. Geomorphology 213 (2014), pp. 166-182, https://doi.org/10. 1016/j.geomorph.2014.01.006

19. Kleppmann, W.: Taschenbuch Versuchsplanung: Produkte und Prozesse optimieren. Praxisreihe Qualitätswissen. München: Hanser, 1998, ISBN: 3-446-19271-9

20. Montgomery, D. C.: Design and analysis of experiments. International student version, 8. edition. [Singapore]: John Wiley \& Sons, 2013, ISBN: 978-1-118-09793-9

21. Siebertz, K.; van Bebber, D.; Hochkirchen, T.: Statistische Versuchsplanung: Design of Experiments (DoE). VDI-Buch. Berlin, Heidelberg: Springer, 2010. ISBN: 978-3-642-05493-8

22. Zhou, Y.; Rupnik, E.; Meynard, C.; Thom, C.; Pierrot-Deseilligny, M.: Simulation and Analysis of photogrammetric UAV Image Blocks: Influence of Camera Calibration Error. ISPRS Annals of Photogrammetry, Remote Sensing and Spatial Information Sciences IV-2/W5 (2019), pp. 195-200. ISSN 2194-9050. https://doi.org/10.5194/isprsannals-IV-2-W5-195-2019

23. Heiss, C.: Überlegungen zur Sicherung von Personen und Infrastrukturbauwerken gegen Steinschlag im alpinen Bereich unter besonderer Berücksichtigung flexibler Steinschlagschutzsysteme - Modellierung flexibler Steinschlagschutzsysteme im Labormaßstab zur Beurteilung nichtgenormter Lastfälle. Diss., Leoben, Montanuniv., Lehrst. F. Bergbaukunde, Bergtechnik und Bergwirtschaft, 2017

24. Moser, P.; Wimmer, M.; Ouchterlony, F.: The fragment size distribution of Kiruna magnetite, from model-scale to run of the mine. In: 5th International Conference and Exhibition on Mass Mining. Luleå Univ. of Technology, 2008, pp. 691-703

25. Agisoft LLC: Agisoft Metashape, 2019. URL: https://www.agisoft. com/ (Zugriff 15.02.2020)

26. Verordnung des Bundesministers für Wirtschaft, Familie und Jugend über Vermessungen beim Bergbau, das Bergbaukartenwerk und die Erfassung von Bodenbewegungen 2013 (Markscheideverordnung 2013), BGBI. II Nr. 437/2012

Hinweis des Verlags. Der Verlag bleibt in Hinblick auf geografische Zuordnungen und Gebietsbezeichnungen in veröffentlichten Karten und Institutsadressen neutral. 